\title{
Preliminary results of entire pleural intensity-modulated radiotherapy in a neoadjuvant setting for resectable malignant mesothelioma
}

\author{
Ji Hyun Hong ${ }^{1}$, Hyo Chun Lee ${ }^{1}$, Kyu Hye Choi', Seok Whan Moon², Kyung Soo Kim², Suk Hee Hong ${ }^{3}$, \\ Ju-Young Hong ${ }^{1}$, Yeon-Sil Kim"; for Multidisciplinary Team of Lung Cancer in Seoul St. Mary's Hospital \\ 'Department of Radiation Oncology, Seoul St. Mary's Hospital, College of Medicine, The Catholic University of Korea, Seoul, Korea \\ ${ }^{2}$ Department of Thoracic and Cardiovascular Surgery, Seoul St. Mary's Hospital, College of Medicine, The Catholic University of \\ Korea, Seoul, Korea \\ ${ }^{3}$ Department of Medical Oncology, Seoul St. Mary's Hospital, College of Medicine, The Catholic University of Korea, Seoul, Korea
}

Purpose: The purpose of this study is to evaluate the safety and efficacy of the multimodality treatment with neoadjuvant intensitymodulated radiotherapy (IMRT) for resectable clinical T1-3N0-1M0 malignant pleural mesothelioma (MPM).

Materials and Methods: A total of eleven patients who received neoadjuvant chemotherapy and radiotherapy between March 2016 and June 2018 were reviewed. Patients received 25 Gy in 5 fractions to entire ipsilateral hemithorax with helical tomotherapy. Results: All of patients were men with a median age of 56 years. Epithelioid subtype was found in 10 patients. All patients received neoadjuvant chemotherapy with pemetrexed-cisplatin regimen. Ten patients (90.9\%) completed $25 \mathrm{~Gy} / 5$ fractions and one (9.0\%) completed $20 \mathrm{~Gy} / 4$ fractions of radiotherapy. IMRT was well tolerated with only one acute grade 3 radiation pneumonitis. Surgery was performed 1 week (median, 8 days; range, 1 to 15 days) after completing IMRT. Extrapleural pneumonectomy was performed in 4 patients (36.3\%), extended pleurectomy/decortication in $2(18.2 \%)$ and pleurectomy/decortications in $5(63.6 \%)$. There was no grade $3+$ surgical complication except two deaths after EPP in 1 month. Based on operative findings and pathologic staging, adjuvant chemotherapy was delivered in 7 patients $(63.6 \%)$, and $2(18.2 \%)$ were decided to add adjuvant radiotherapy. After a median follow-up of 14.6 months (range, 2.8 to 30 months), there were 3 local recurrence (33.3\%) and 1 distant metastasis (11.1\%).

Conclusion: Neoadjuvant entire pleural IMRT can be delivered with a favorable radiation complication. An optimal strategy has to be made in resectable MPM patients who would benefit from neoadjuvant radiation and surgery. Further studies are needed to look at long-term outcomes.

Keywords: Mesothelioma, Neoadjuvant therapy, Radiotherapy, Combined modality therapy, Hemithoracic radiation

\section{Introduction}

Malignant pleural mesothelioma (MPM) is an aggressive cancer derived from the pleural surface. MPM is an uncommon malignancy, with an incidence of 14,200 cases worldwide annually [1]. The incidence of MPM usually peaks and declines

Received 05 March 2019, Revised 15 April 2019, Accepted 23 May 2019.

Correspondence: Yeon-Sil Kim, Department of Radiation Oncology, Seoul St. Mary's Hospital, College of Medicine, The Catholic University of Korea, 222 Banpo-daero, Seocho-gu, Seoul 06591, Korea. Tel: +82-2-2258-6259, Fax: +82-2-2258-1532, E-mail: yeonkim7@catholic.ac.kr (http://orcid.org/0000-0003-1291-0821)

(c) This is an Open Access article distributed under the terms of the Creative Commons Attribution Non-Commercial License (http://creativecommons.org/ licenses/by-nc/4.0/) which permits unrestricted non-commercial use, distribution, and reproduction in any medium, provided the original work is properly cited.

www.e-roj.org 


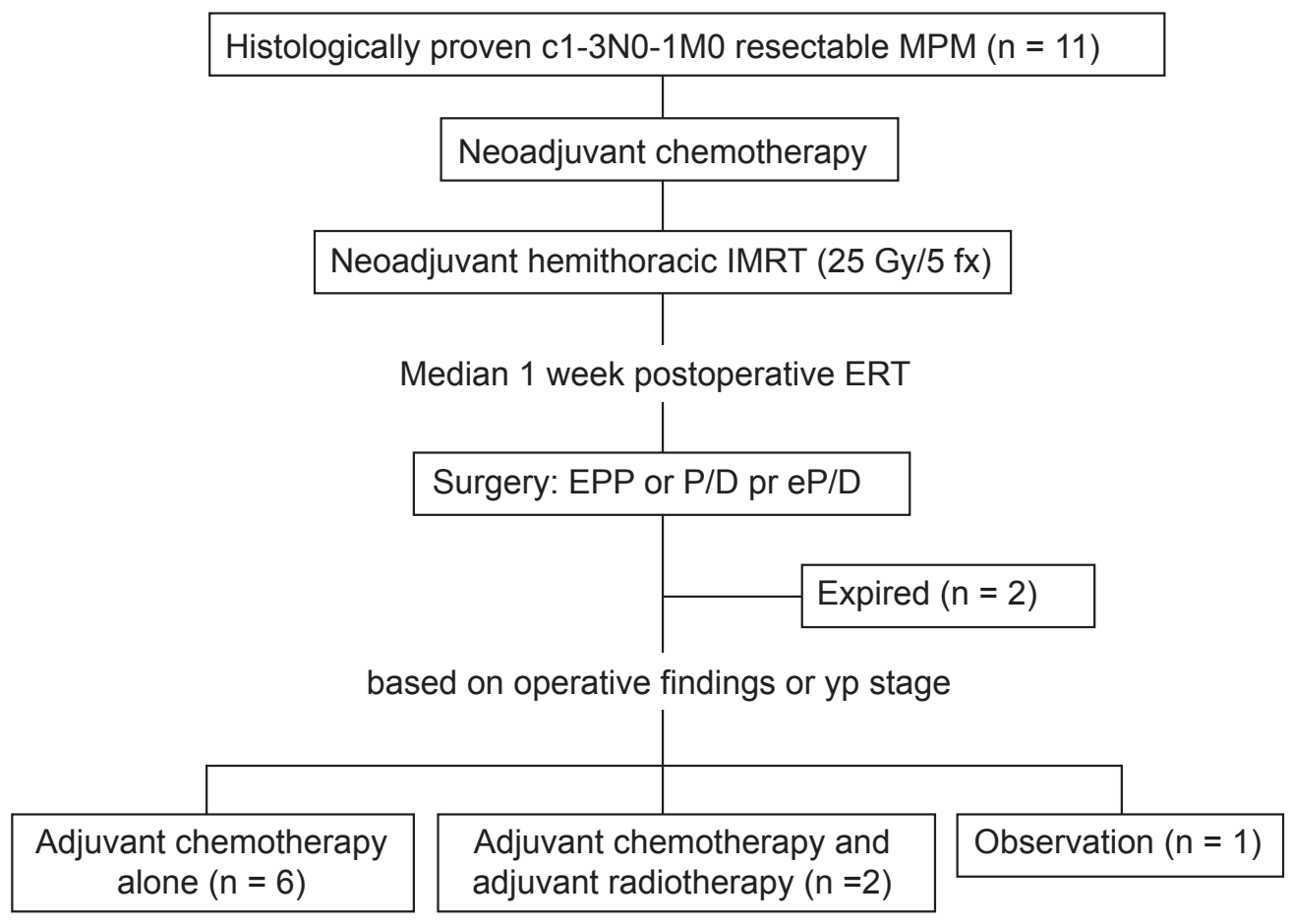

Fig. 1. Surgery for Mesothelioma After Radiation Therapy (SMART) protocol in our institution. MPM, malignant pleural mesothelioma; IMRT, intensity-modulated radiotherapy; ERT, emergency response team; EPP, extrapleural pneumonectomy; P/D, pleurectomy/ decortication; EP/D, extended pleurectomy/decortication.

20-30 years after the use of asbestoses. In Korea, the asbestos industry began in the 1960s, and the industry peaked in the 1990s. In 2009, the use of asbestos was banned. Therefore, the incidence of MPM is expected to increase until 2045 [2].

Despite its increasing incidence, there is no consensus on the best treatment for MPM. The treatment of MPM is very challenging, and its overall prognosis is poor with a 2-year survival of $0 \%-12 \%$ [3]. Although there have been various attempts to find an appropriate treatment from the best conservative care to multimodality treatments, the outcomes remain dismal [4]. As some studies have shown that surgical resection plays an important role in multimodality treatment, the optimal treatment for surgically resectable MPM has attracted attention [5].

Based on the study from the Princess Margaret Cancer Centre (PM) and Toronto General Hospital (TGH), our institution developed a new protocol with induction chemotherapy, a short accelerated course of high-dose hypofractionated entire pleural intensity-modulated radiotherapy (IMRT) followed by surgery $[3,6,7]$. Our protocol, called the Surgery for Mesothelioma After Radiation Therapy (SMART), is only for resectable MPM with clinical T1-3N0-1 without distant metastasis. In this study, the short-term outcomes and safety of the protocol were evaluated.

\section{Materials and Methods}

\section{Patients}

Between March 2016 and June 2018, 15n patients with MPM received radiotherapy $(\mathrm{RT})$ at our institution. Four patients were excluded from the analysis since the intent of treatment was not preoperative. Eleven patients treated with the SMART protocol were included in this study (Fig. 1). All patients were discussed among a multidisciplinary team to decide their enrollment in the SMART protocol. Each patient was diagnosed with malignant mesothelioma with histological confirmation via either video-assisted thoracoscopic surgery biopsy or chest wall soft tissue biopsy. All tumors were staged using the American Joint Committee on Cancer (AJCC) 8th edition and defined surgically resectable as T1-3N0-1 without distant metastasis [8]. None of the patients had a history of RT or other cancer treatments. This study was approved by the Institutional Review Board of Seoul St. Mary's Hospital (No. KC18RESI0737).

\section{SMART protocol in our institution}



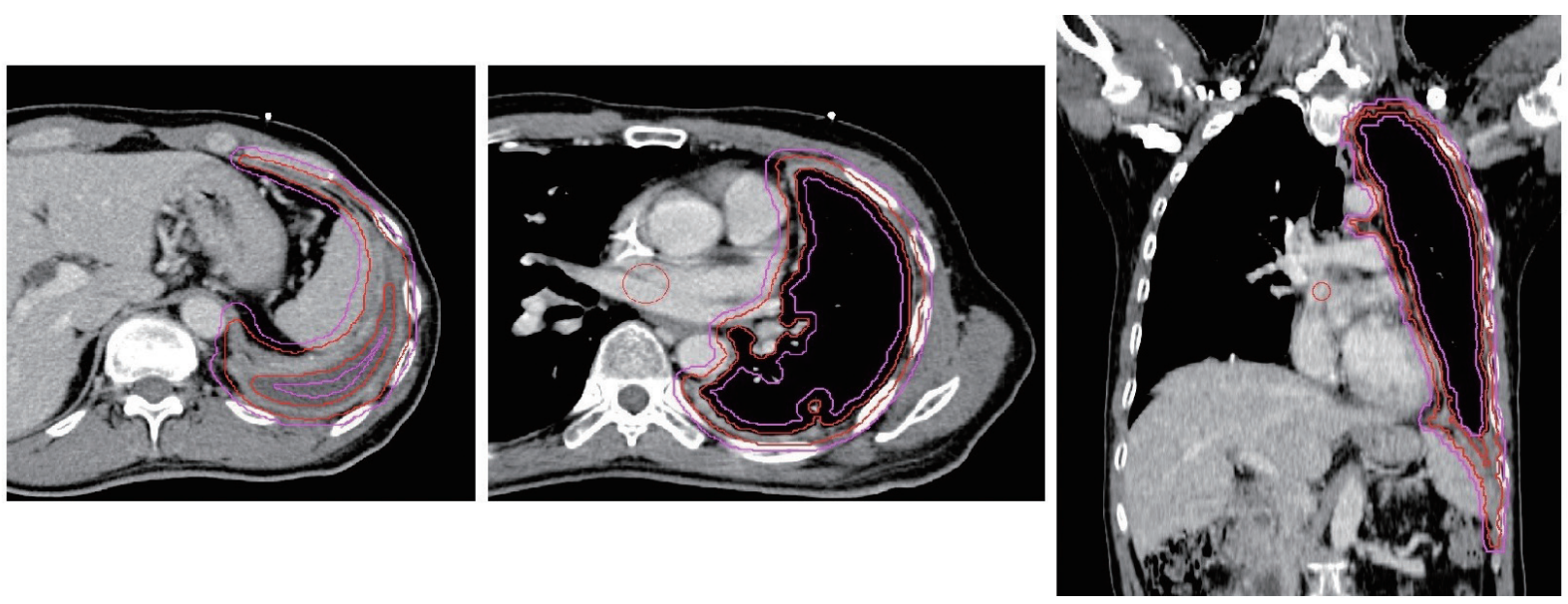

Fig. 2. Target delineation of the entire pleura with two axial views and a coronal view. Clinical target volume (CTV, red line) and planning target volume (pink line) were generated by CTV $+3-5 \mathrm{~mm}$.

The SMART protocol in our institution includes neoadjuvant chemotherapy, short-course radiation therapy to the entire ipsilateral hemithorax followed by curative surgery. Surgery is performed within 2 weeks of completing RT. After the surgery, all patients were discussed in a multidisciplinary team. Patients who underwent pleurectomy/decortication (P/D) or extended pleurectomy/decortication (EP/D) decided to be treated with adjuvant chemotherapy, and those who underwent extrapleural pneumonectomy (EPP) were determined on the final pathological and surgical findings (Fig. 1).

\section{Neoadjuvant chemotherapy}

All patients received at least two cycles of neoadjuvant chemotherapy consisting of $500 \mathrm{mg} / \mathrm{m}^{2}$ pemetrexed and 75 $\mathrm{mg} / \mathrm{m} 2$ cisplatin on day 1 (D1). Seven patients received two cycles of cisplatin-pemetrexed before RT. One patient received three cycles and two patients received four cycles, since the differences between the initial images and images following two cycles of chemotherapy did not allow the surgeon to decide upon surgical resectability. One patient visited our institution after being treated with six cycles of cisplatinpemetrexed at another institution. The patient received five additional cycles of carboplatin and gemcitabine before RT: $1,000 \mathrm{mg} / \mathrm{m}^{2}$ gemcitabine on D1 and D8 and $450 \mathrm{mg} / \mathrm{m}^{2}$ carboplatin on D1.

\section{Radiotherapy - target delineation}

All patients were immobilized with a Vac-Lock and underwent four-dimensional (4D) planning computed tomography (CT) for IMRT using Tomotherapy. Gross tumor volume included the gross tumor seen on the CT or fluorodeoxyglucose- positron emission tomography (FDG-PET) image. Clinical target volume (CTV) was defined as the entire ipsilateral hemithorax, including suspicious lymph nodes. The parietal pleura along the ribs and mediastinal pleura along the pericardium were included from the superior thoracic inlet to the lowest level of diaphragm insertion. Since entire ipsilateral hemithoracic cage was irradiated, movements depending on the locations were different. Considering movements based on $4 \mathrm{D}-\mathrm{CT}$, and possible toxicities of hypofractionated RT using 5 Gy fraction on organs close to diaphragm such as liver, kidney, stomach and heart, narrow planning target volume (PTV) was practically decided 3-5 mm margin from CTV (Fig. 2). The prescribed dose to the PTV was 25 Gy in 5 fractions over 1 week.

\section{Surgery}

Surgery included EPP, EP/D, and P/D. EPP removes the parietal and visceral pleura, including the ipsilateral lung, pericardium and adjacent diaphragm, with reconstruction of the pericardium and diaphragm. P/D removes the affected pleura without the pericardium and/or diaphragm, whereas EP/D removes the affected pleura along with the pericardium and/ or diaphragm [9]. Thoracic surgeons decided on the method of operation based on the condition of the patient or imaging studies. Furthermore, hyperthermia using hot water infusion and photodynamic therapy were included during surgical procedures.

\section{Follow-up and response assessment}

After completing the treatment course, patients were regularly followed up at 2-3 month intervals with chest $\mathrm{CT}$, abdomen $C T$, chest X-rays, blood tests, and FDG-PET if needed. Local 
Table 1. Patients' characteristics

\begin{tabular}{|c|c|}
\hline Characteristic & Value \\
\hline Age (yr) & $56(36-69)$ \\
\hline \multicolumn{2}{|l|}{ Sex } \\
\hline Male & $11(100)$ \\
\hline Female & $0(0)$ \\
\hline \multicolumn{2}{|l|}{ Laterality } \\
\hline Right & 7 (63.6) \\
\hline Left & $4(36.4)$ \\
\hline \multicolumn{2}{|l|}{ Presentation } \\
\hline Symptomatic & $10(90.9)$ \\
\hline Incidental & $1(9.1)$ \\
\hline \multicolumn{2}{|l|}{ Histology } \\
\hline Epithelioid & $10(90.9)$ \\
\hline Biphasic & $1(9.1)$ \\
\hline \multicolumn{2}{|l|}{ PreRT PFT } \\
\hline FEV1 (L) & $2.41(2.06-3.01)$ \\
\hline DLCO (\%) & $64.0(46.0-113.0)$ \\
\hline \multicolumn{2}{|l|}{ Clinical T stage } \\
\hline $\mathrm{T} 1$ & $3(27.3)$ \\
\hline $\mathrm{T} 2$ & $3(27.3)$ \\
\hline T3 & $5(45.5)$ \\
\hline \multicolumn{2}{|l|}{ Clinical N stage } \\
\hline No & $6(54.5)$ \\
\hline N1 & $5(45.5)$ \\
\hline \multicolumn{2}{|l|}{ Response after NACTx } \\
\hline PR & $3(27.3)$ \\
\hline SD & 8 (72.7) \\
\hline \multicolumn{2}{|l|}{ Operation } \\
\hline EPP & $4(36.4)$ \\
\hline$E P / D$ & $2(18.2)$ \\
\hline$P / D$ & $5(45.5)$ \\
\hline
\end{tabular}

Values are presented as median (range) or number (\%).

$\mathrm{RT}$, radiotherapy; PFT, pulmonary function test; FEV1, forced expiratory volume in 1 second; $\mathrm{DLCO}$, diffusing capacity of the lungs for carbon monoxide; NACTx, neoadjuvant chemotherapy; $P R$, partial response; SD, stable disease; EPP, extrapleural pneumonectomy; EP/D, extended pleurectomy and decortications; $P / D$, pleurectomy and decortications.

recurrence was defined as the appearance of recurrence within the ipsilateral chest. Recurrences were diagnosed clinically by serial imaging with or without pathologic confirmation. Responses were evaluated according to the Response Evaluation Criteria in Solid Tumors (RECIST) 1.1. Toxicities were evaluated by the Common Terminology Criteria for Adverse Events (CTCAE) version 4.03.

\section{Statistical analysis}

Descriptive statics were reported, and continuous data are presented as the mean or median values. Survival outcomes were estimated using the Kaplan-Meier analysis. Survival was calculated from the day of diagnosis for all patients. Overall survival (OS) was defined as the time between the day of diagnosis and the date of the death or the most recent followup visit. Local progression-free survival (LPFS) and distant metastasis-free survival (DMFS) were defined as the time between the day of diagnosis and the date of recurrence or the most recent follow-up visit or death when there was no recurrence. All statistical analyses were performed with SPSS version 23.0 (IBM Corp., Armonk, NY, USA).

\section{Results}

\section{Patient characteristics}

After a multidisciplinary evaluation, 11 patients were enrolled in the protocol. Patient characteristics are summarized in Table 1. Most patients initially presented with dyspnea (90.9\%) with pleural effusion (54.5\%). One patient visited a clinic with an abnormal chest finding from a regular checkup.

The median age of patients was 56 years, and all patients were male. The tumor was located on the right hemithorax in 7 patients (63.6\%) and on the left in the others. The patients' pulmonary function tests were good, with a median forced expiratory volume in 1 second (FEV1) of $2.41 \mathrm{~L}$ (range, 2.06 to $3.01 \mathrm{~L}$ ) and a median value of diffusing capacity of the lungs for carbon monoxide (DLCO) of 64.0\% (range, 46.0\% to $113.0 \%$ ). The clinical stage was T3N1MO and T2NOMO in 3 patients, respectively, T3NOMO and T1N1M0 in 2 patients each, and T1NOMO in 1 patient.

Three patients (27.3\%) showed partial remission after only two cycles of neoadjuvant cisplatin-pemetrexed chemotherapy. Eight patients (72.7\%) showed stable disease.

All patients completed the SMART protocol, including chemotherapy, radiation therapy, and curative surgery. No patient dropped out during the course of the protocol. EPP with macroscopically complete resection of the tumor was performed in 4 patients (36.4\%), EP/D was performed in 2 patients (18.2\%), and P/D was performed in 5 patients (45.5\%). Surgery was performed a median of 8 days (range, 1 to 15 days) after the completion of RT. There were two (18.2\%) 90day mortalities after EPP. One patient expired after 31 days because of sudden arrhythmia, and the other expired after 16 days due to aspiration pneumonia.

The final histology of tumors was epithelioid type in 10 patients (90.9\%) and biphasic type in 1 patient (9.1\%). Two patients were diagnosed with pN2 in the final pathology, and 
Table 2 . Summary of organ at risk dose

\begin{tabular}{|c|c|c|}
\hline Organs at risk & SMART protocol of PMH & Dose of current study \\
\hline Mean lung dose (cGy) & - & $1,042.6(804-1,303.6)$ \\
\hline Ipsilateral lung mean dose (cGy) & - & $1,943.3(1,662.5-2117)$ \\
\hline Contralateral lung mean dose (cGy) & $<350$ & $403.9(268.9-539.1)$ \\
\hline Contralateral lung $V_{7}(\%)$ & $<20$ & $4.2(0.4-19.2)$ \\
\hline Mean heart dose (cGy) & - & $1,308.5(816-1,693.1)$ \\
\hline Rt. MPM, heart $V_{15}(\%)$ & $<40$ & $32.5(12.6-41.6)$ \\
\hline Lt. MPM, heart $V_{18}(\%)$ & $<70$ & $29.85(21-42.6)$ \\
\hline Mean liver dose (cGy) & - & $1,045.6(525.9-1,580.7)$ \\
\hline Rt. MPM, liver $\mathrm{V}_{17}(\%)$ & $<60$ & $33.4(17.8-45.9)$ \\
\hline Lt. MPM, liver $\bigvee_{8}(\%)$ & $<30$ & $20.8(15.7-27)$ \\
\hline Ipsilateral kidney mean dose (cGy) & a) & $587.3(245.9-1,354.4)$ \\
\hline Contralateral kidney mean dose (cGy) & a) & $268.8(87.5-544.4)$ \\
\hline Esophagus mean dose (cGy) & - & 1,133.6 (871.4-1,640.1) \\
\hline Esophagus maximum dose (cGy) & $<3,000$ & $2,331.2(1,938.2-2,642.2)$ \\
\hline Spinal cord maximum dose (cGy) & $<2,200$ & $1,731.7(1,138.7-2,352.8)$ \\
\hline
\end{tabular}

Values are presented as mean (min-max).

SMART, Surgery for Mesothelioma After Radiation Therapy; PMH, the Princess Margaret Hospital; $V_{n}$, percentage of the planning target volume receiving $n \%$ of the prescription dose; MPM, malignant pleural mesothelioma.

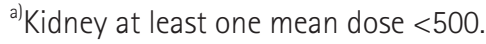

Table 3. Complications after the protocol (CTCAE v4.03)

\begin{tabular}{|c|c|c|c|c|c|c|}
\hline Complication & Total (\%) & Grade 1 & Grade 2 & Grade 3 & Grade 4 & Grade 5 \\
\hline $\begin{array}{l}\text { Patients with grade } 3+\text { complications } \\
\text { RT complication }\end{array}$ & $6(55)$ & - & - & - & - & - \\
\hline Radiation pneumonitis & $4(36)$ & 1 & 2 & 1 & - & - \\
\hline Radiation esophagitis & $2(18)$ & 1 & 1 & - & - & - \\
\hline Loss of appetite & $3(27)$ & 2 & 1 & - & - & - \\
\hline \multicolumn{7}{|l|}{ Operation complication } \\
\hline Pneumonia & $2(18)$ & - & - & 1 & - & 1 \\
\hline Wound infection & 1 (9) & - & - & 1 & - & - \\
\hline Chest wall fistula & $1(9)$ & 1 & - & - & - & - \\
\hline Skin burna) & $1(9)$ & 1 & - & - & - & - \\
\hline Hemothorax & $1(9)$ & - & - & 1 & - & - \\
\hline Arrhythmia & $1(9)$ & - & - & - & - & 1 \\
\hline
\end{tabular}

CTCAE, Common Terminology Criteria for Adverse Events; RT, radiotherapy.

a) Photosensitive dermatitis due to photodynamic therapy and intrapleural hyperthermia

1 patient, whose stage was cT3NOMO, was pT4N1M0. Two patients who died within 90 days of surgery and one who had been treated with more than six cycles of chemotherapy at another institution did not receive additional adjuvant treatment. Except for these 3 patients, 7 patients were treated with adjuvant cisplatin-pemetrexed chemotherapy, and 1 patient who underwent $P / D$, and 1 patient who showed local recurrence were treated with adjuvant $\mathrm{RT}$.

\section{Target volume and organ at risk mean dose}

The mean PTV was 2,264.9 $\mathrm{mL}$ (range, 1,368.2 to 4,409.6 mL), and the mean CTV was $1,067.9 \mathrm{~mL}$ (range, 388.5 to $2,900.8$ $\mathrm{mL}$ ). The contralateral, ipsilateral and total lung, heart, liver, both kidneys, spinal cord and esophagus were contoured as organs at risk (OAR). The mean lung dose (MLD) was $10.4 \mathrm{~Gy}$ (range, 8.04 to $13.04 \mathrm{~Gy}$ ), and the MLD of the contralateral lung was $4.04 \mathrm{~Gy}$ (range, 2.69 to $5.39 \mathrm{~Gy}$ ). Doses to the OAR were tried to be constrained as the SMART protocol of PM and 


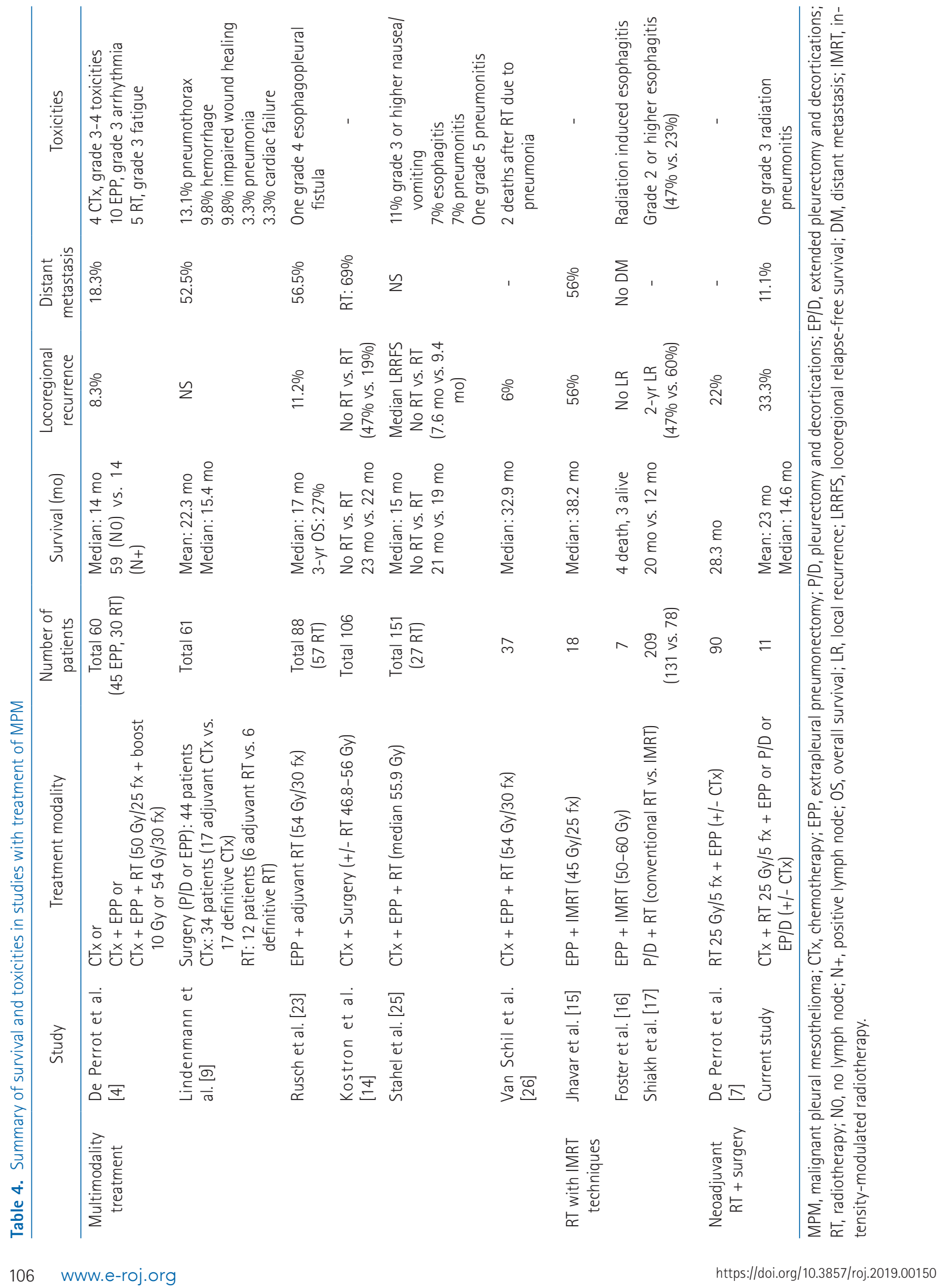


TGH [7] (Table 2), and the mean value of the dose to the OAR is listed in Table 2.

\section{Survival and pattern of failure}

The median follow-up was 14.6 months (range, 2.8 to 30.0 months). The mean OS was 23.0 months and median OS was 14.6 months. A total of 3 patients (27.3\%) expired, and all of them died without any evidence of recurrence. Three patients (33.3\%) developed recurrence in the ipsilateral pleura of the thoracic cage at 5.5 months, 8.1 months, and 10.6 months from the operation date. One patient who showed local recurrence 8.1 months after the operation developed multiple lung nodules in the contralateral lung parenchyma and multiple enlarged lymph nodes in the retroperitoneum after 2 additional months (10.5 months after surgery). No other patient showed distant metastasis. The mean LPFS was 14.8 months, and the mean DMFS was 17.9 months. The EORTC score [10], including pleural involvement, lactate dehydrogenase level, performance status, platelet count, histology and age, and CALGB score [11], including PS, white blood cell count, histology and gender, did not show any significant difference in survival.

\section{Toxicity}

Six patients (54.5\%) developed higher than grade 3 complications. Four patients had radiation pneumonitis. Among them, one patient who showed grade 3 radiation pneumonitis was treated with steroids after hospitalization. In spite of hypofractionated neoadjuvant radiation therapy, fistula did not occur after surgery. There were five patients with higher than grade 3 surgical complications, which included three grade 3 and two grade 5 complications (Table 3 ).

\section{Discussion and Conclusion}

The treatment of MPM has generally been the best conservative care with a poor median survival ranging between 6 and 8 months [12]. Systemic chemotherapy with pemetrexed plus cisplatin, which is the only US Food and Drug Administrationapproved therapy for MPM, extends the median survival to 12 to 16 months. However, the trimodality treatment of induction chemotherapy, EPP or P/D, and adjuvant hemithoracic radiation therapy has resulted in improved survival outcomes, with a median survival time of more than 20 months [13]. One study achieved a median survival of 59 months in patients with ypNO disease who finished all multimodality treatments [4].

https://doi.org/10.3857/roj.2019.00150
Since local recurrence remains the most frequent type of relapse after treatment, RT plays a role in improving local control. Kostron et al. [14] analyzed the outcomes of patients treated with induction chemotherapy and surgery. In the previous study, patients who received adjuvant RT showed significantly less local recurrence than patients who underwent surgery alone ( $19 \%$ vs. $47 \%$, respectively; $p=0.003$ ). Furthermore, developing radiation techniques impacts survival and toxicity outcomes. The IMRT technique makes it possible to deliver a desired dose of radiation that is highly conformal to the target and spares normal tissues by improving dose distribution and dose homogeneity $[13,15,16]$. Jhavar et al. [15] reported good survival outcomes by treatment with IMRT after EPP with a median survival of 38.2 months. Shiakh et al. [17] evaluated the outcomes of IMRT compared with conventional RT, and concluded that IMRT improved OS (median, 20.2 months vs. 12.3 months) with less toxicity such as esophagitis (Table 4).

However, as trimodality treatment has a long treatment period and is an aggressive treatment, administering adjuvant hemithoracic radiation is so difficult that only half of the patients can complete the treatment of all three modalities [4]. Moreover, approximately 25\% of patients showed disease progression during induction chemotherapy, and afterwards, they were precluded from EPP. Furthermore, distant recurrence was still frequent (69\%) after trimodality treatment [14].

Based on the results of a previous study, SMART protocol of our institution contains three steps: induction chemotherapy to reduce distant failure with less aggressiveness than upfront surgery; a short accelerated course of high-dose hypofractionated hemithoracic radiation therapy to control local recurrence, preventing patients from dropping out from a long period of treatment; and, shortly after RT, surgery. Differently from SMART protocol of PM and MGH, our protocol included patients with clinically N1 disease, contained neoadjuvant chemotherapy, and underwent surgical approaches other than EPP. Adjuvant chemotherapy was considered based on the findings from surgery or previous chemotherapy through a multidisciplinary team. With our SMART protocol, no patient dropped out over the treatment course. Considering the median 1-week interval between RT and surgery, RT was given not to downstage the tumor but to induce a tumorostatic effect of the tumor to prevent tumor spillage during the operation, and to activate the immune system against the tumor with hypofractionated radiation $[6,18]$. The rationale for our short and intense course of chest RT combined with chemotherapy prior to surgery is to sterilize 
tumor cells in the thorax and to avoid or reduce the spread to areas outside of the chest cavity. This approach may reduce the incidence of locoregional recurrence and metastasis in these aggressive diseases, ultimately improving survival.

Optimal surgical approaches to MPM remain controversial. EPP and EP/D are the most commonly used surgical techniques with curative intent [19]. However, EPP results in significant complications and mortality [20]. Verma et al. [21] reported no significant difference between EPP and P/D in OS (19 months vs. 16 months, respectively; $p=0.12$ ), 30-day mortality (5\% vs. $5 \%$, respectively; $p=0.999)$, or 30 -day readmission rate $(7 \%$ vs. $5 \%$, respectively; $p=0.292$ ). Therefore, our multidisciplinary team allowed the thoracic surgeon to choose the surgical technique based on imaging, previous therapy, and the patient's condition.

SMART protocol, pioneered by the PM and TGH with Dr. de Perrot chose EPP as the surgical technique to prevent the risk of pneumonitis, since there is a risk of severe pneumonitis after surgical techniques such as P/D and EP/D $[3,6,7,19]$. They treated 90 patients between 2008 and 2017 and reported favorable outcomes with a median survival of 28.3 months and acceptable toxicities [7]. There was $22 \%$ locoregional recurrence, which was defined by recurrence along the ipsilateral thorax, and histological subtype, nodal disease, tumor thickness and tumor volume were identified as significant factors on the outcome. The epithelial subtype showed the most favorable outcome. The median survival for the epithelial subtype with tumor volumes less than $500 \mathrm{~mL}$ was 51 months.

Although this study included surgical techniques other than EPP, there was no radiation pneumonitis greater than grade 4 , except for one patient who underwent P/D that showed grade 3 radiation pneumonitis. Compared with other studies using adjuvant high-dose hemithoracic irradiation greater than 40 Gy with grade 3 radiation pneumonitis of 0\%-20\% [7,22-24], only one radiation pneumonitis (9\%) was considered to have tolerable toxicity. After induction chemotherapy, all patients were able to be administered radiation therapy and surgery, since there was no progressive disease in our study. The survival outcomes were comparable with other studies. Local recurrence occurred in 3 patients (33.3\%) in our study, while other studies have reported locoregional recurrence rates after trimodality treatment of 16\%-89\% $[7,25,26]$.

This study has limitations, such as a short follow-up period and a small number of patients. Because of the rarity of this disease, it is difficult to build a protocol in a single institution. A longer follow-up and a larger number of patients are needed to statistically analyze the outcomes. In addition, heterogeneities of surgical methods, and adjuvant chemotherapy are our shortcomings. Toxicity after P/D, EP/D and EPP would be different due to the remains of normal lung tissue, and differences in adjuvant chemotherapy might affect survival outcomes after treatment. Nevertheless, this approach is encouraging and a leading strategy that could be used to treat surgically resectable MPM in Korea.

In the era of immunotherapy, there are ongoing studies and clinical trials. Immune checkpoint inhibitors have variable efficacies, and studies for developing a therapy combining immunotherapy and chemotherapy are currently ongoing $[12,27,28]$. Additionally, de La Maza et al. [18] reported that hypofractionation can activate the immune system. A study combining immunotherapy with previous treatments, such as chemotherapy, surgery, and radiation, is also expected to have promising outcomes.

In conclusion, a neoadjuvant short accelerated course of high-dose hypofractionated hemithoracic IMRT can be feasible with favorable radiation complications. Optimal patient selection must be performed in resectable MPM patients. Further studies are needed to look at long-term outcomes, and experience with the SMART protocol will increase over time.

\section{Conflict of Interest}

No potential conflict of interest relevant to this article is reported.

\section{References}

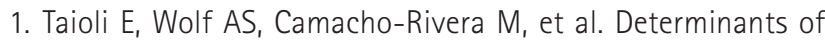
survival in malignant pleural mesothelioma: a Surveillance, Epidemiology, and End Results (SEER) study of 14,228 patients. PLoS One 2015;10:e0145039.

2. Kwak KM, Paek D, Hwang SS, Ju YS. Estimated future incidence of malignant mesothelioma in South Korea: Projection from 2014 to 2033. PLoS One 2017;12:e0183404.

3. Cho BC, Feld R, Leighl $N$, et al. A feasibility study evaluating Surgery for Mesothelioma After Radiation Therapy: the "SMART" approach for resectable malignant pleural mesothelioma. J Thorac Oncol 2014;9:397-402.

4. de Perrot $M$, Feld $R$, Cho BC, et al. Trimodality therapy with induction chemotherapy followed by extrapleural pneumonectomy and adjuvant high-dose hemithoracic radiation for malignant pleural mesothelioma. J Clin Oncol 2009;27:1413-8. 
5. Nelson DB, Rice DC, Niu J, et al. Long-term survival outcomes of cancer-directed surgery for malignant pleural mesothelioma: propensity score matching analysis. J Clin Oncol 2017;35:3354-62.

6. de Perrot M, Feld R, Leighl NB, et al. Accelerated hemithoracic radiation followed by extrapleural pneumonectomy for malignant pleural mesothelioma. J Thorac Cardiovasc Surg 2016;151:468-73.

7. de Perrot $M, W u L, W u$ M, Cho BC. Radiotherapy for the treatment of malignant pleural mesothelioma. Lancet Oncol 2017;18:e532-e542.

8. Abdel-Rahman 0. Challenging a dogma; AJCC 8th staging system is not sufficient to predict outcomes of patients with malignant pleural mesothelioma. Lung Cancer 2017;113:12833.

9. Lindenmann J, Matzi V, Neuboeck N, et al. Multimodal therapy of malignant pleural mesothelioma: is the replacement of radical surgery imminent? Interact Cardiovasc Thorac Surg 2013;16:237-43.

10. Curran D, Sahmoud T, Therasse $P$, van Meerbeeck J, Postmus $P E$, Giaccone G. Prognostic factors in patients with pleural mesothelioma: the European Organization for Research and Treatment of Cancer experience. J Clin Oncol 1998;16:145-52.

11. Herndon JE, Green MR, Chahinian AP, Corson JM, Suzuki Y, Vogelzang NJ. Factors predictive of survival among 337 patients with mesothelioma treated between 1984 and 1994 by the Cancer and Leukemia Group B. Chest 1998;113:723-31.

12. McCambridge AJ, Napolitano A, Mansfield AS, et al. Progress in the management of malignant pleural mesothelioma in 2017. J Thorac Oncol 2018;13:606-23.

13. Chi A, Liao Z, Nguyen NP, et al. Intensity-modulated radiotherapy after extrapleural pneumonectomy in the combined-modality treatment of malignant pleural mesothelioma. J Thorac Oncol 2011;6:1132-41.

14. Kostron A, Friess $M$, Crameri 0 , et al. Relapse pattern and second-line treatment following multimodality treatment for malignant pleural mesothelioma. Eur J Cardiothorac Surg 2016;49:1516-23.

15. Jhavar S, Pruszynski J, Gowan A, Boyle T, Deb N, Patel M. Intensity modulated radiation therapy after extra-pleural pneumonectomy for malignant pleural mesothelioma is feasible without fatal pulmonary toxicity and provides good survival. Asia Pac J Clin Oncol 2018;14:e88-e94.

16. Forster KM, Smythe WR, Starkschall G, et al. Intensitymodulated radiotherapy following extrapleural pneumonectomy for the treatment of malignant mesothelioma: clinical implementation. Int J Radiat Oncol Biol
Phys 2003;55:606-16.

17. Shaikh F, Zauderer MG, von Reibnitz D, et al. Improved outcomes with modern lung-sparing trimodality therapy in patients with malignant pleural mesothelioma. J Thorac Oncol 2017;12:993-1000.

18. De La Maza $L, W u M, W u$, et al. In situ vaccination after accelerated hypofractionated radiation and surgery in a mesothelioma mouse model. Clin Cancer Res 2017;23:550213.

19. Murphy DJ, Gill RR. Overview of treatment related complications in malignant pleural mesothelioma. Ann Transl Med 2017:5:235.

20.Kao SC, Cheng YY, Williams M, et al. Tumor suppressor microRNAs contribute to the regulation of PD-L1 expression in malignant pleural mesothelioma. J Thorac Oncol 2017;12:1421-33.

21. Verma V, Ahern CA, Berlind CG, et al. National Cancer Database report on pneumonectomy versus lung-sparing surgery for malignant pleural mesothelioma. J Thorac Oncol 2017;12:1704-14.

22. Gupta $V$, Mychalczak B, Krug L, et al. Hemithoracic radiation therapy after pleurectomy/decortication for malignant pleural mesothelioma. Int J Radiat Oncol Biol Phys 2005;63:1045-52.

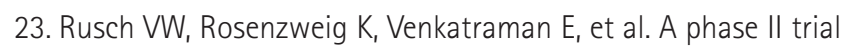
of surgical resection and adjuvant high-dose hemithoracic radiation for malignant pleural mesothelioma. J Thorac Cardiovasc Surg 2001;122:788-95.

24. Kimura T, Doi Y, Nakashima $T$, et al. Clinical experience of volumetric modulated arc therapy for malignant pleural mesothelioma after extrapleural pneumonectomy. J Radiat Res 2015;56:315-24.

25. Stahel RA, Riesterer 0 , Xyrafas $A$, et al. Neoadjuvant chemotherapy and extrapleural pneumonectomy of malignant pleural mesothelioma with or without hemithoracic radiotherapy (SAKK 17/04): a randomised, international, multicentre phase 2 trial. Lancet Oncol 2015;16:1651-8.

26. Van Schil PE, Baas $P$, Gaafar $R$, et al. Trimodality therapy for malignant pleural mesothelioma: results from an EORTC phase II multicentre trial. Eur Respir J 2010;36:1362-9.

27. Alley EW, Katz SI, Cengel KA, Simone CB 2nd. Immunotherapy and radiation therapy for malignant pleural mesothelioma. Transl Lung Cancer Res 2017;6:212-9.

28. Tang C, Welsh JW, de Groot $P$, et al. Ipilimumab with stereotactic ablative radiation therapy: phase I results and immunologic correlates from peripheral T cells. Clin Cancer Res 2017;23:1388-96. 